\title{
Experimental Analysis of Gas-Sensitive Braitenberg Vehicles
}

\author{
Achim Lilienthal ${ }^{1}$ and Tom Duckett ${ }^{2}$ \\ ${ }^{1}$ W.-Schickard-Inst. for Comp. Science, University of Tübingen, Sand 1, 72074 Tübingen, Germany \\ 2 Department of Technology, AASS, Örebro University, S-70182 Örebro, Sweden
}

\begin{abstract}
This article addresses the problem of localising a static gas source in an indoor environment by a mobile robot. In contrast to previous works, the environment is not artificially ventilated to produce a strong unidirectional airflow. Here, the dominant transport mechanisms of gas molecules are turbulence and convection flow rather than diffusion, which results in a patchy, chaotically fluctuating gas distribution. Two Braitenberg-type strategies (positive and negative tropotaxis) based on the instantaneously measured spatial concentration gradient were investigated. Both strategies were shown to be of potential use for gas source localisation. As a possible solution to the problem of gas source declaration (the task of determining with certainty that the gas source has been found), an indirect localisation strategy based on exploration and concentration peak avoidance is suggested. Here, a gas source is located by exploiting the fact that local concentration maxima occur more frequently near the gas source compared to distant regions.
\end{abstract}

\section{Introduction}

Using electrochemical sensors on a mobile robot is very promising for a broad range of applications. A robot equipped with gas sensors could be utilised as an electronic watchman that is able to detect, localise and identify odours, perhaps indicating toxic gas leaks, leaking solvents or a fire at its initial stage. Furthermore self-produced odours can be used to aid navigation [5] or to communicate with other robots, for instance by sending a chemical SOS signal [30].

Localising a gas source in real world environments is a challenging task mainly because the distribution of odourant molecules is usually dominated by turbulence rather than diffusion. As a consequence, the resulting gas distribution is patchy and chaotic, and the actual gas source is usually not located at the point of highest intensity. Investigating the problems that arise from this situation can lead to a deeper understanding of the physical properties of turbulent motion, as well as the way in which animals use odours for navigation purposes.

\subsection{Reactive Localisation Strategies}

Localisation behaviours in biology often involve reactive components that determine control commands only from the instantaneously sensed properties of the stimulus field [7]. Such behaviours do not require long-term memory because no explicit representation of the environment is needed. Consequently, biological evolution tends to favour these minimalistic solutions if they are sufficient to solve the given task. The minimal capabilities needed for reactive localisation are 
- a mechanism to extract directional information from the sensed signal (tracing), and

- the ability to determine whether the source has been found (declaration).

This article addresses both issues while it starts by investigating the question to what extent reactive strategies are sufficient for gas source tracing in an uncontrolled indoor environment. This problem was investigated by means of a mobile robot governed towards a single immovable gas source. The robot was steered as a Braitenberg vehicle [2] using a direct sensor-motor coupling based on the instantaneously measured spatial concentration gradient (pure chemotaxis).

\subsection{Gas Source Tracing by Gradient-Following}

Unlike visual or auditory stimuli, chemical stimuli are not inherently directional. In order to achieve spatial chemo-orientation an animal therefore has to determine a concentration gradient from comparing either successive stimuli (klinotaxis) or simultaneously sensed intensities from two or more receptors (tropotaxis). As pointed out by Grasso in Holland and McFarland [12], the traditional framework for understanding animal orientation by Fraenkel and Gunn [7] is limited because it assumes a uniform gradient of the distribution. This assumption is usually not fulfilled for gas distributions in natural environments. Due to the low diffusion velocity of gases at room temperature [27], the dispersal of an analyte gas is dominated by turbulence and the prevailing air flow rather than diffusion [36]. A real gas distribution therefore reveals many discontinous patches of local eddies [11,29], thus creating local concentration maxima that can mislead a gradient following strategy. Moreover, the absolute maximum of the instantaneous distribution is usually not located near the gas source if this source has been active for some time [21].

Considering the chaotic nature of turbulent gas transport, the applicability of reactive gas source tracing techniques that follow the instantaneously measured spatial gradient seems arguable. Due to the smooth course of the averaged distribution, however, following the gradient towards the stronger signal should be capable of driving a robot toward a gas source on average. The question to what degree this is true is the starting point of this investigation.

\subsection{Related Work}

Most work on chemical sensing for mobile robots assumes an experimental setup that minimizes the influence of turbulent transport by either minimising the source-to-sensor distance in trail following [28,33-35] or by assuming a strong unidirectional airstream in the environment $[10,13,30,32]$. Primarily a strong airstream can be used to get additional information about the local wind speed and direction from an anemometer. Thus, strategies become feasible that utilise the instantaneous direction of flow as an estimate of source direction [3] by combining gas searching behaviours with periods of upwind movement. Furthermore, the condition of a constant unidirectional airstream implies a more structured distribution of the analyte. For such situations it is possible to model the time-averaged spread of gas [11] because the complexity of turbulent air movement can be described 


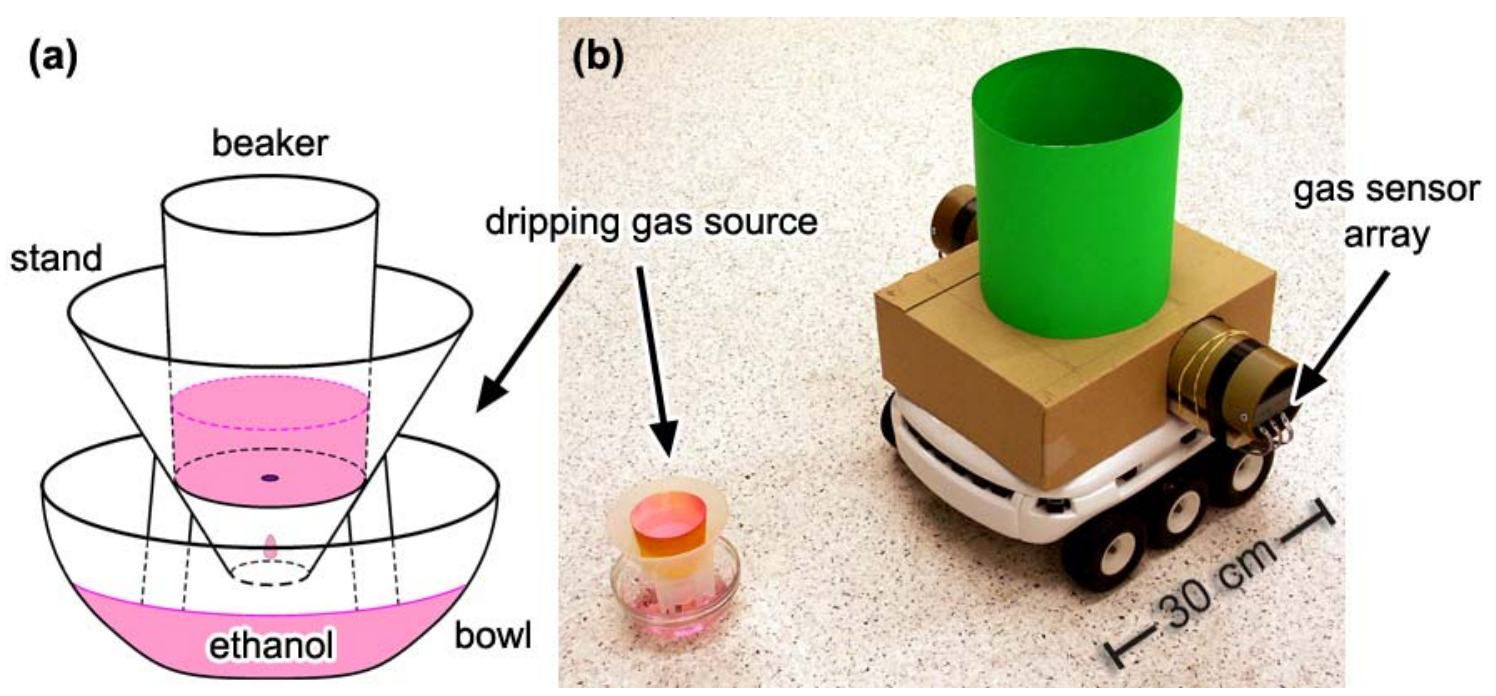

Fig. 1. Koala robot with the Örebro Mark III mobile nose and the dripping gas source used in the experiments. The gas source is schematically shown on the left side (a), and the robot is depicted in front of the gas source on the right side (b).

with a diffusion-like behaviour ruled by an additional diffusion coefficient (see [14]). The available wind measuring devices, however, are limited in their applicable range. With state-of-the-art anemometers based on the cooling of a heated wire [14], the bending of an artificial whisker [31] or the influence on the speed of a small rotating paddle [30], reliable readings can be obtained only for wind speeds in the order of at least $10 \mathrm{~cm} / \mathrm{s}$.

The intention of this work is to enable a mobile robot to trace a gas source and declare whether the source has been found without being restricted to an environment with a strong unidirectional airflow (see also [4, 6, 24, 25]).

The rest of this article is structured as follows: first, the experimental setup and the experiments performed are described in Sections 2 and 3. The corresponding experimental results are then discussed in Section 4 followed by conclusions and suggestions for future work (Section 5).

\section{Experimental Setup}

\subsection{Robot and Gas Sensors}

The experiments were performed with a Koala mobile robot (see Fig. 1) equipped with the Mark III mobile nose [18], comprising 6 tin oxide sensors manufactured by Figaro. This type of chemical sensor shows a decreasing resistance in the presence of reducing volatile chemicals in the surrounding air. In consequence of the measurement principle, metal oxide sensors exhibit some drawbacks, including the low selectivity, comparatively high power consumption (caused by the heating device) and weak durability. In addition, metal oxide sensors are subject to a long response time and an even longer recovery time. The time 
constants of rise and decay for the complete gas sensitive system (mobile nose) used here were estimated as $\tau_{r} \approx 1.8 \mathrm{~s}$ and $\tau_{d} \approx 11.1 \mathrm{~s}$ respectively [18]. However, this type of gas sensor is most often used on mobile robots because it is inexpensive, highly sensitive and relatively unaffected by changing environmental conditions such as room temperature and humidity. The sensors were placed in sets of three (of type TGS 2600, TGS 2610 and TGS 2620) inside two separate tubes containing a suction fan each. An array of redundant sensors was used only to increase the robustness of the system (there was no attempt to discriminate different gases by pattern classification, as in most traditional studies using non-mobile electronic noses [9]). Papst Fans (405F) were used to generate an airflow of approximately $8 \mathrm{~m}^{3} / \mathrm{h}$. The distance between the two sets of sensors was $40 \mathrm{~cm}$.

\subsection{Absolute Positioning System}

To record the position of the robot, the vision-based absolute positioning system $\mathrm{W}$ CAPS [19] was applied, which tracks a distinctly coloured object mounted on top of the robot (the cardboard "hat" shown in Fig. 1). The positioning system uses four Philips PCVC $740 \mathrm{~K}$ web-cameras mounted at fixed positions with a resolution of $320 \times 240$ pixels to triangulate the $(\mathrm{x}, \mathrm{y})$ position of the centre of the colour blob. By combining up to 6 single position estimates, it provides centimeter level accuracy. Fig. 2 shows the camera positions and the respective fields of view. The graded shadings indicate the number of cameras that can sense each part of the environment.

The heading $\vartheta$ of the robot can be estimated from the tangent to the robot's path while the robot is moving at non-zero speed. By fusing these estimates with the values provided by odometry, an accurate estimate of the heading is obtained that is not subject to long term drift. Further details are given in [19].

\subsection{Environment and Gas Source}

All experiments were performed in a rectangular laboratory room at Örebro University (size $\left.10.6 \times 4.5 \mathrm{~m}^{2}\right)$. The robot's movement was restricted so that its centre was always located inside the central region where precise and reliable position information is available. The air conditioning system in the room was deactivated in order to eliminate the possibility of a dominant constant airflow.

To emulate a typical task for an inspection robot, a gas source was chosen to imitate a leaking tank. This was realised by placing a paper cup filled with ethanol on a support in a bowl with a perimeter of $12 \mathrm{~cm}$ (see Fig. 1). The ethanol dripped through a hole in the cup into the bowl at a rate of approximately $50 \mathrm{ml} / \mathrm{h}$. Ethanol was used because it is non-toxic and easily detectable by the tin oxide sensors.

\section{Experiments}

\subsection{Braitenberg Vehicles}

Two different tropotaxis behaviours that use a direct sensor-motor coupling were considered. This kind of steering architecture is known as a Braitenberg vehicle (see Fig. 3) due to 


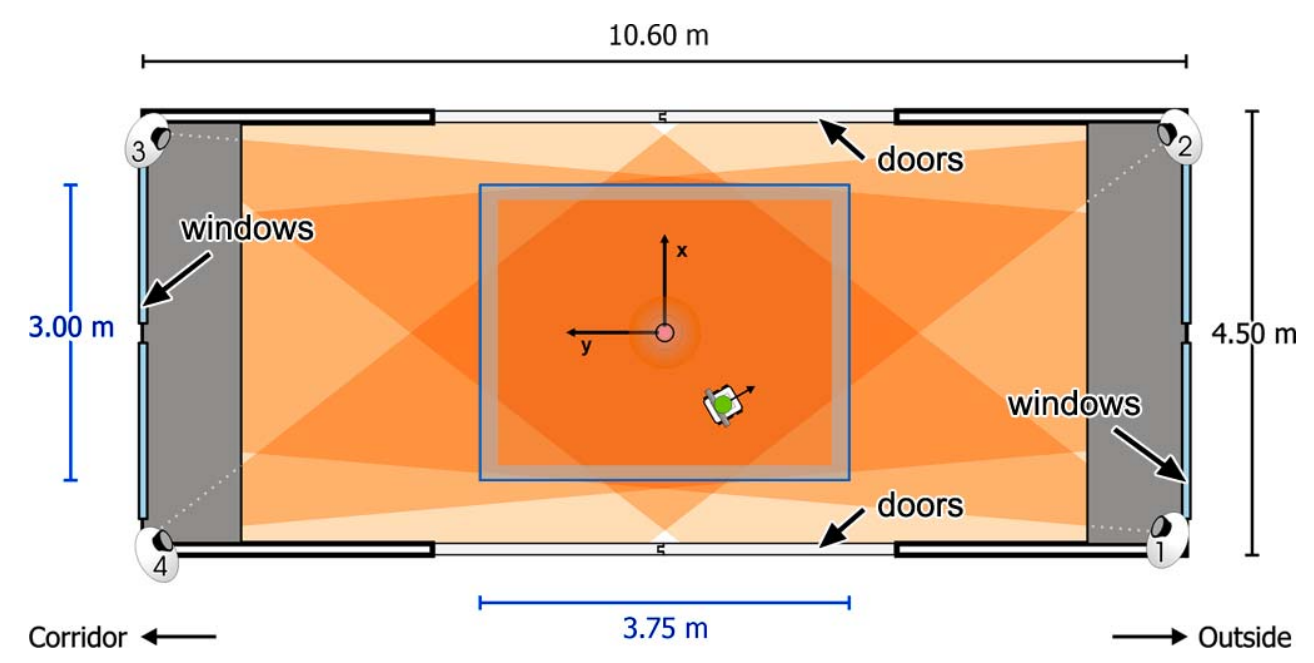

Fig. 2. Testbed area for gas source tracing experiments. The figure shows a floor plan of the laboratory room (including doors and windows) and the outline of the region in which the experiments were performed. Additionally, the repellent potential field is indicated at the edge of the testbed area. Also plotted are the fields of view for each of the four cameras that were used to track the robot's position, shaded according to the number of cameras which can sense a particular region.

the influential thought experiments of Valentino Braitenberg [2]. In his book Braitenberg explains which kind of behaviour should result for these vehicles (denominated as type 2, 3 and 4) using different classes of intermediate transfer functions $v(x)$ and assuming a uniform gradient. For all the experiments presented here, inhibitory connections that apply a monotonic transfer function were used. Thus, maximum wheel speed results if the sensed concentration is low, which in turn implements a simple sort of exploration behaviour. On the other hand the robot is slowed down by high concentrations of the analyte.

With uncrossed connections (Fig. 3, left) and a monotonic, inhibitory transfer function, the wheel on the side that is stimulated more is driven slower and therefore the robot turns to this side. This behaviour was called "permanent love" by Braitenberg because it is supposed to move the vehicle to a source of stimulation and stay near this source in theory. Note that "high concentration" or "stimulation" in this context always means "high sensor values" and that these values do not reflect the actual concentration directly due to the non-zero response and the strong memory effect caused by the long recovery time of the metal oxide sensors, as discussed in Section 2.1.

With crossed connections (Fig. 3, right) and an inhibitory, monotonic transfer function the robot is also slowed down by elevated sensor responses but will in contrast turn away from them. Such a vehicle tends to stay at locations near to a maximum of stimulation, too, but continues to wander if another maximum comes into focus. Accordingly, Braitenberg called this kind of behaviour "exploring love". Again this description applies to a system with ideal sensors that moves guided by a smooth distribution peaked just at the actual location of any gas sources. 

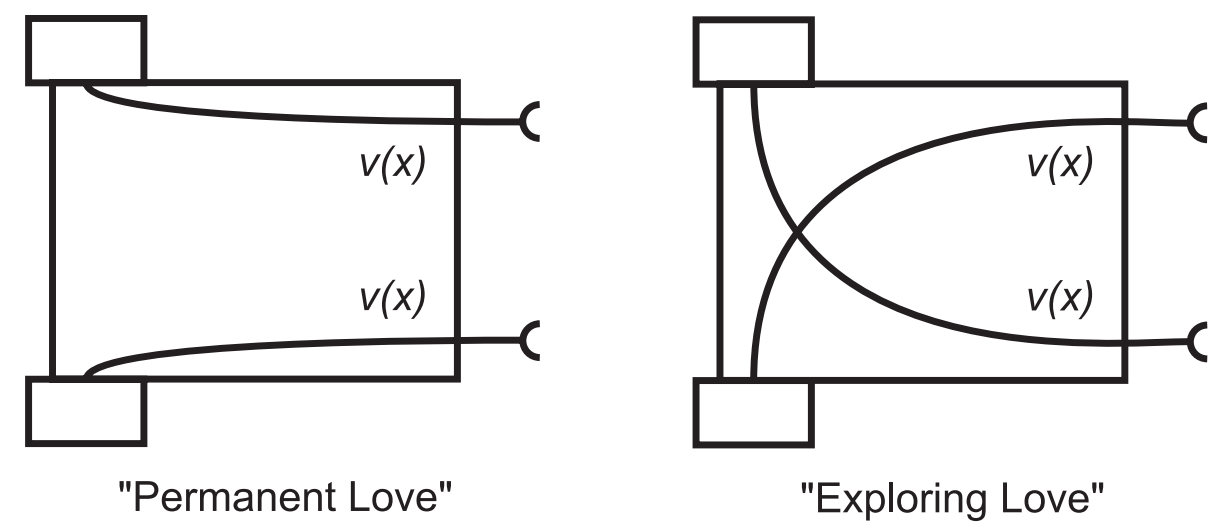

Fig. 3. Schematic view of Braitenberg vehicles with a direct inhibitory sensor motor coupling. Left: uncrossed connections ("permanent love"). Right: crossed connections ("exploring love").

\subsection{Sensor Preprocessing}

The sensor-motor wiring realises a transfer function $v(x)$ that determines the speed of the connected wheel from the sensed quantity $x$. This quantity is supposed to represent the concentration of the analyte at the location of the corresponding sensor. In order to determine the relationship between the raw sensor readings and the corresponding absolute concentration values, calibration of each sensor is necessary because the response characteristics of individual sensors differ considerably. Calibrating gas sensors, however, is a costly operation because it has to be performed under well-defined laboratory conditions. Moreover, the whole process would have to be repeated frequently in order to compensate for the long term drift of metal oxide gas sensors. Finally, the environmental conditions, including temperature, humidity and the local airflow would have to be precisely measured to determine the absolute concentration with a robot.

On this account, standard calibration methods [1] would be not suitable for the given task. Instead, the value $x$ is calculated by normalising the raw sensor readings $r_{i}$ to lie within the range $[0,1]$. For this purpose, the minimum and maximum value of the raw sensor readings $r_{\text {min,i }}$ and $r_{\max , i}$ are constantly updated for each sensor $i$ and used to calculate the normalised response $x_{i}$ as

$$
x_{i}^{(t)}=\frac{r_{i}^{(t)}-r_{m i n, i}^{(t)}}{r_{\max , i}^{(t)}-r_{\min , i}^{(t)}} .
$$

Then, the normalised response values belonging to one side of the robot are combined by averaging as

$$
x_{L}^{(t)}=\sum_{i=1}^{N_{L}} x_{i}^{(t)} / N_{L}, \quad x_{R}^{(t)}=\sum_{i=1}^{N_{R}} x_{i}^{(t)} / N_{R},
$$

where $N_{L}$ and $N_{R}$ are the number of sensors contained in the sensor array on the left and right side respectively. For the experiments presented, $N_{L}=N_{R}=3$ sensors were used. 
Because it eliminates the need for external intervention, such a normalisation technique provides a practical solution for possible applications, which is able to compensate for the sensitivity mismatch of individual sensors as well as for seasonal and environmental drifts. Rather than the absolute concentration of the analyte, the normalised value $x$ represents the relative concentration with respect to the current situation ${ }^{3}$.

While it is possible to compensate for different conditions in individual runs, the normalisation technique described so far is unable to adapt to varying conditions during a single trial. In response to a changing temperature, for example, the normalisation range gets wider and might not cover the actual range of values with time. This causes changes in response to be less pronounced in $x$. Note that the same effect occurs naturally if the average concentration is rising due to the presence of an active gas source. To avoid this problem, the normalisation range is dynamically trimmed each $\Delta t^{\text {trim }}$ seconds by increasing the minimum and decreasing the maximum value in Eq. 1 by a fixed fraction of the normalisation range as

$$
\begin{aligned}
& r_{\min , i}^{(t)}=\tilde{r}_{\min , i}^{(t)}+\Delta_{\min }^{\operatorname{trim}}\left(\tilde{r}_{\max , i}^{(t)}-\tilde{r}_{\min , i}^{(t)}\right), \\
& r_{\max , i}^{(t)}=\tilde{r}_{\max , i}^{(t)}-\Delta_{\max }^{\operatorname{trim}}\left(\tilde{r}_{\max , i}^{(t)}-\tilde{r}_{\min , i}^{(t)}\right) .
\end{aligned}
$$

Here, $\tilde{r}_{\min , i}^{(t)}$ and $\tilde{r}_{\max , i}^{(t)}$ refer to the minimum and maximum value at time $t$ before trimming.

Consequently, the normalised value $x$ represents the relative concentration with respect to the concentration extremes that occur at that time more closely. In all the experiments, the trimming parameters

$$
\Delta_{\min }^{\text {trim }}=\Delta_{\max }^{\text {trim }}=1 \% \text { and } \Delta t^{\text {trim }}=30 \mathrm{~s}
$$

were used, which were found to be suitable in an initial test sequence.

\subsection{Transfer Function}

In all experiments presented, the following linear inhibitory transfer function was used:

$$
v(x)=K_{v}(1-x)
$$

with a velocity gain $K_{v}$ of either $5 \mathrm{~cm} / \mathrm{s}$ or $3 \mathrm{~cm} / \mathrm{s}$.

\subsection{Testbed Scenario}

Both Braitenberg-type strategies considered, which can be also described as "exploration and hillclimbing" (Fig. 3, left) and "exploration and concentration peak avoidance" (Fig. 3, right), were tested repeatedly with the following scenario. A $3.75 \mathrm{~m} \times 3 \mathrm{~m}$ field was defined by establishing virtual walls. These boundaries were realised by assigning an artificial potential field [16] that effects a repellent pseudo-force, which increases linearily with the

\footnotetext{
${ }^{3}$ Note that the assumption of a linear resistance-concentration dependence is made here.
} 
penetration depth and starts to be effective at a distance of $20 \mathrm{~cm}$. The experiments were performed in the central region of the room where precise and reliable position information is available (see Fig. 2).

Now the robot could move freely within this virtual field while being constantly tracked by the absolute positioning system. Next, a gas source was placed at a known position inside the field. Then a series of experiments was performed with this configuration as follows:

- set the robot to a random starting position inside the virtual field (with a clearance of at least $100 \mathrm{~cm}$ to the centre of the source),

- rotate the robot to a random initial heading,

- start to move the robot with the particular control strategy under investigation,

- count a successful trial and restart if the robot enters the obstacle clearance area around the gas source.

These steps were repeated for a fixed amount of time while the position and the sensor readings were constantly logged for evaluation purposes. For the reference experiments, no gas source was used. However, "successful" trials were counted when the robot entered the clearance area around a dummy obstacle that was placed inside the field instead of an active gas source.

\section{Results}

The following sections present a discussion of the behaviour of gas-sensitive Braitenberg vehicles with uncrossed ("permanent love") and crossed inhibitory connections ("exploring love"). Typical trajectories are shown in Figures $4-7$. Here, the path of the robot's centre is indicated by a hollow circle, while the position of the front corners is plotted using small dots. The starting position and the initial heading of the corresponding trial are marked by an arrow, which originates from the starting position. Also shown are the virtual repellent walls (broken line) that enclose the area where the repellent force increases with the penetration depth of the robot. Finally, the clearance area of the gas source is shown by two circles. A trial was stopped if one of the front corners of the robot entered the inner circle. The outer circle was derived by obstacle growing [17], assuming a circular shaped robot. The radius was chosen to be the minimum distance between the centre of the robot and the source. Note that because the robot actually has a rectangular profile, the outer circle provides just an approximate notion of the obstacle's boundary with respect to the centre of the robot.

\subsection{Interpretation Using Concentration Gridmaps}

In order to explain the resulting trajectories, it would be helpful to know the transient gas distribution at each time step. Due to the local limitation of single gas sensor measurements, this is not achievable without covering the whole available area with a dense grid of sensors. Besides other problems, such an array of metal oxide sensors would cause a severe disturbance due to the convective flow created by the heaters built into these sensors [15]. 

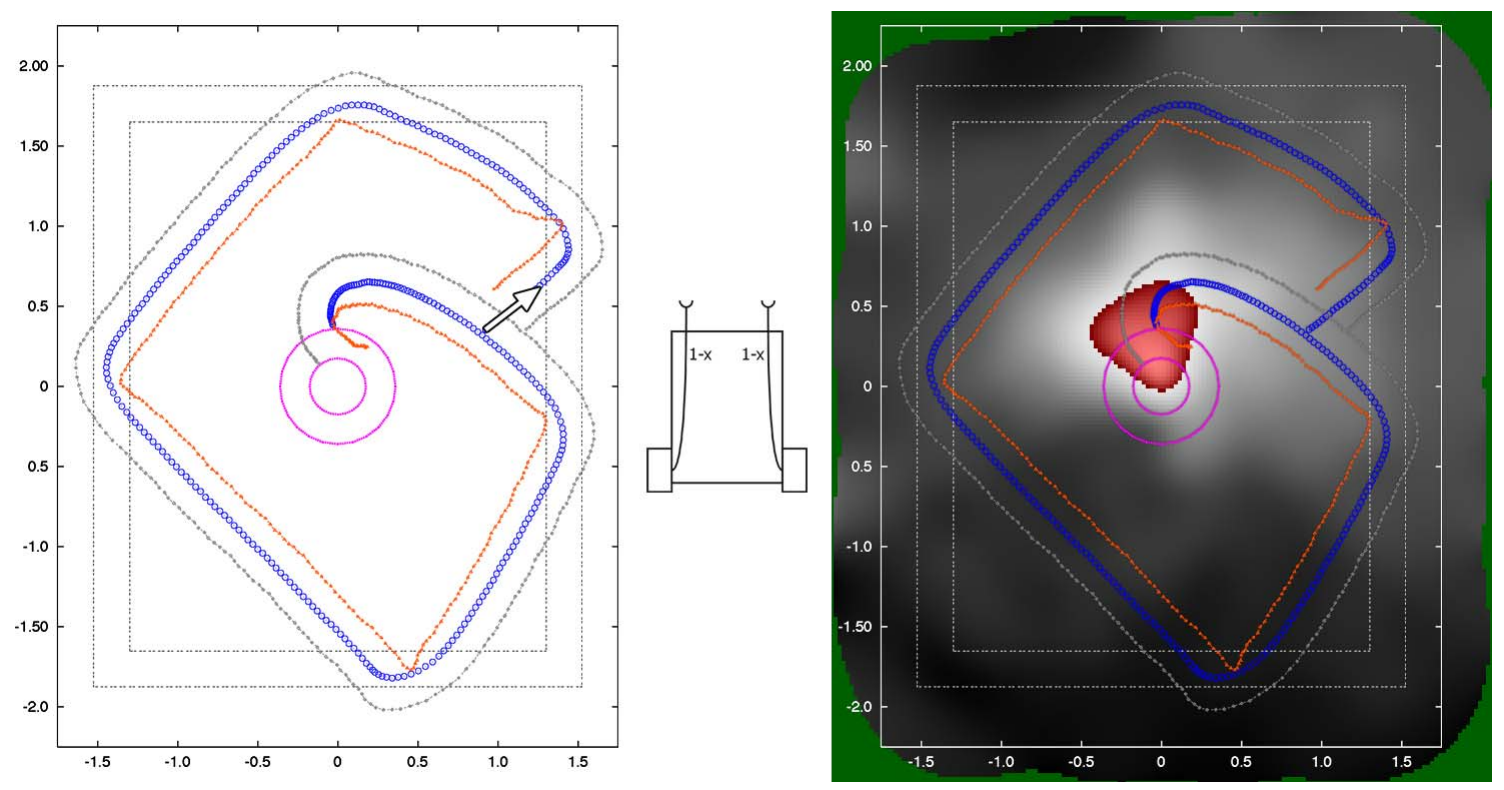

Fig. 4. Example of the path of a "permanent love" Braitenberg vehicle (uncrosssed (1-x)-connections) with the gas source in the middle of the testbed area.

However, while the transient distribution cannot be measured with the gas sensors carried by the robot, its time-invariant structure can be determined using the technique to create gridmaps of the relative gas concentration introduced in [20].

In addition to the typical trajectories shown on the left side of Figures $4-7$, the same trajectory is shown on the right side on top of the corresponding gas concentration gridmap. These gridmaps were created offline with a cell size of $2.5 \times 2.5 \mathrm{~cm}^{2}$ from the gas sensor readings recorded during all the trials belonging to one experiment. Limited by the available battery capacity, complete experiments lasted approximately 3 hours. To illustrate these gridmaps, the relative concentration values are indicated by shadings of grey (dark $\rightarrow$ low, light $\rightarrow$ high). In order to represent plume-like structures, values higher than 90\% of the maximum are plotted with a second range of dark-to-light shadings. Further details concerning gas concentration mapping are given in [20,22].

\subsection{Localisation by Exploration and Hillclimbing}

In the case of uncrossed connections ("permanent love"), the robot could frequently reach the source in a strikingly straightforward way, as in the example trial shown in Fig. 4. In this experiment the source was placed in the middle of the virtual field. The robot was almost unaffected by the gas source until it bumped into the virtual wall for the fifth time. As can be seen on the right side of Fig. 4, the behaviour before the fifth collision is consistent with the concentration gridmap, as the robot remained outside the region of high concentration during that time. Then, the robot entered the region of highest concentration (the plume), 

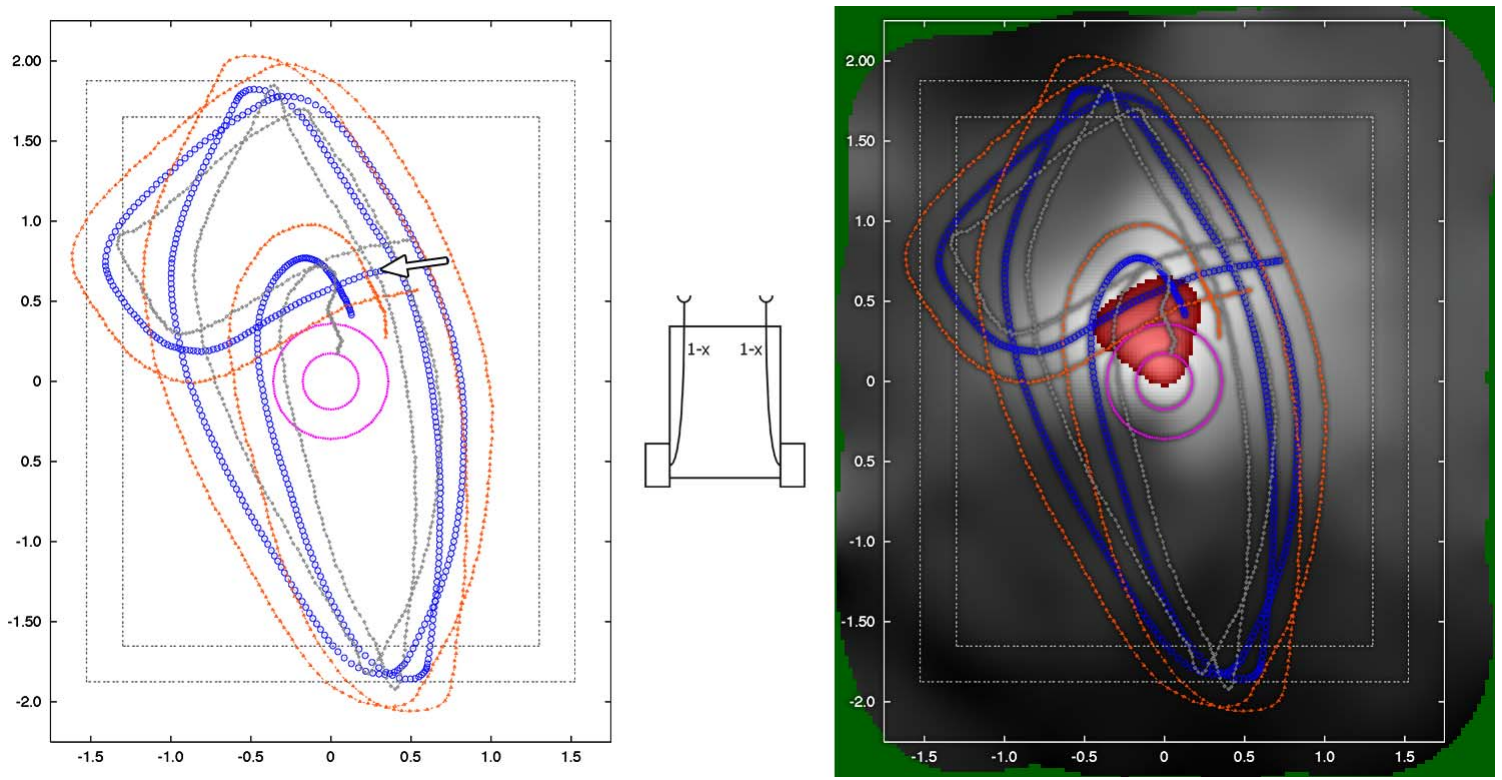

Fig. 5. Example of the path of a "permanent love" Braitenberg vehicle (uncrosssed (1-x)-connections) with the gas source in the middle of the testbed area.

slowed down, turned to the left side (where it had entered the plume first), and finally hit the clearance area around the gas source.

While the previously mentioned trial could be explained in terms of the average distribution, this is not always possible. The trajectory shown in Fig. 5, for example, is likely to be caused by transient concentration fluctuations. This trial was performed directly after the one shown in Fig. 4. Here, the robot passed the gas source immediately after the trial was started at a very low distance. However, hardly any reaction was obtained, neither concerning the translational nor the rotational speed. The path during the remainder of the trial appears consistent with the gas concentration map. The robot orbited around the gas source and moved along roughly straight lines outside the region of high concentration. Ultimately the robot entered the plume (with the right side first), turned back and slowed down, and finally moved slowly towards the source.

The statistical evaluation of the experiments, with the gas source placed in the middle of the testbed area, is summarised in Table 1. Here, the leftmost column specifies the position of the gas source while the second column refers to the applied strategy, denoted as Ref (reference experiment), PL (uncrossed connections, "permanent love") or EL (crossed

\begin{tabular}{|c|cccc|ccc|}
\hline Source & Strategy & $K_{v}\left[\frac{c m}{s}\right]$ & Trials & $l_{\text {tot }}[\mathbf{m}]$ & $\bar{l}[\mathbf{m}]$ & $\bar{d}[\mathbf{c m}]$ & $\bar{v} / K_{v}$ \\
\hline \multirow{2}{*}{ Middle } & Ref $(1-\mathrm{x})$ & 5 & 33 & 319.0 & $9.67 \pm 7.66$ & $136.7 \pm 44.9$ & $95.9 \%$ \\
& PL, 1-x & 5 & 123 & 1044.0 & $8.49 \pm 7.93$ & $127.4 \pm 45.7$ & $73.5 \%$ \\
\hline
\end{tabular}

Table 1. Statistics of the gas source tracing experiments with a Braitenberg vehicle and the source placed in the middle of the testbed area. Comparison of the "permanent love" behaviour (PL) with the reference strategy (Ref). 

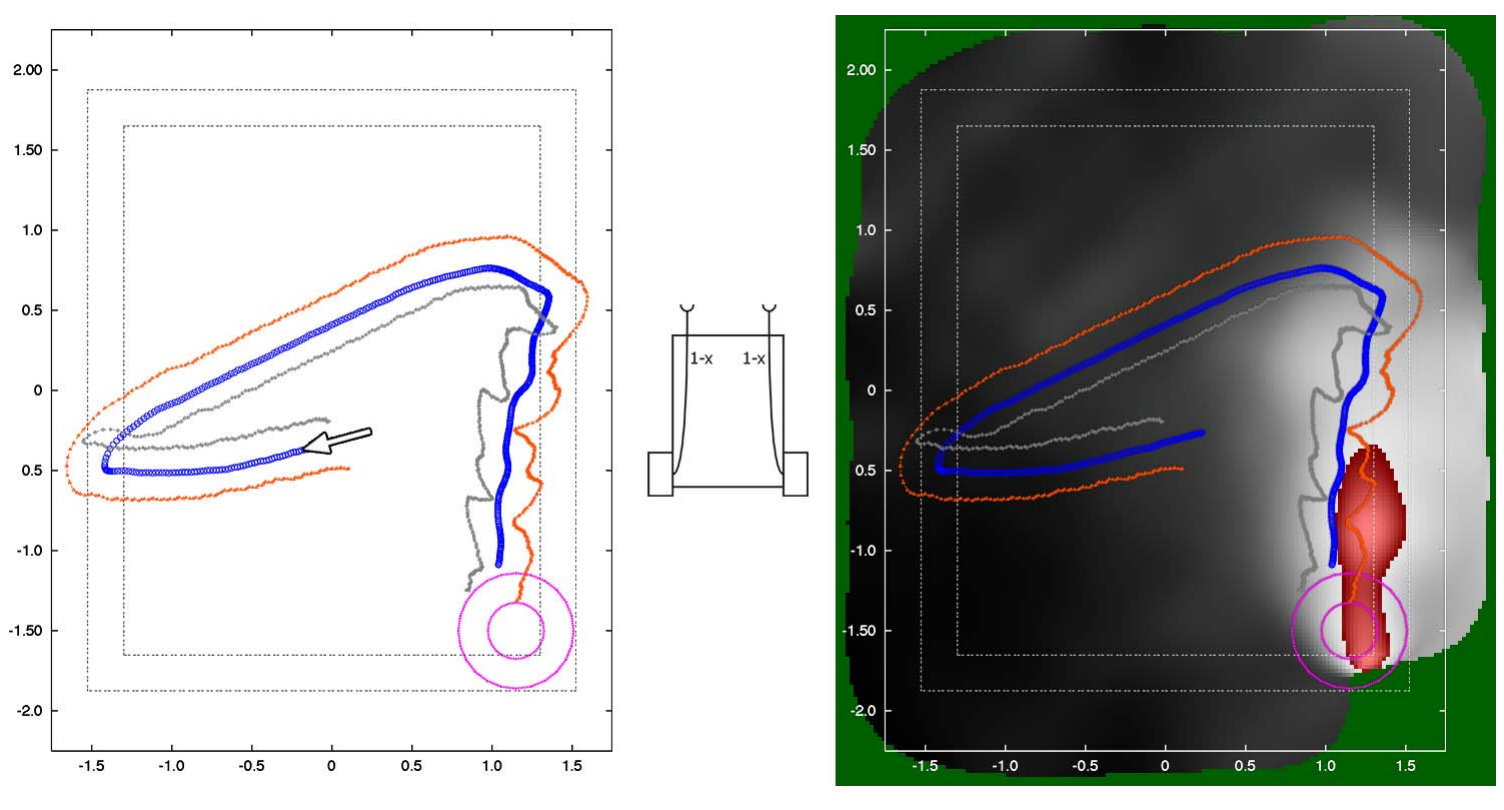

Fig. 6. Example of the path of a "permanent love" Braitenberg vehicle (uncrosssed (1-x)-connections) with the gas source in a corner of the testbed area.

connections, "exploring love"). The remaining columns itemise (from left to right): the speed gain $K_{v}$, the number of trials where the robot entered the clearance area ("successful trials"), the total path length $l_{\text {tot }}$, the average path length of successful trials $\bar{l}$, the average distance to the source $\bar{d}$, and the average velocity $\bar{v}$ in units of the speed gain. To validate the results, reference tests were performed without a gas source present. Because the sensor readings were not considered during these tests, the robot moved basically like a ball on a billiard table. As in all other experiments, a successful trial was counted when the robot entered the area assigned to be the source.

With the source placed in the middle of the inspected area, the average path length of a vehicle with uncrossed (1-x)-connections was $8.49 \mathrm{~m}$ compared to $9.67 \mathrm{~m}$ for the reference (the median was $6.57 \mathrm{~m}$ compared to $8.31 \mathrm{~m}$ ). Applying the Wilcoxon two sample test ${ }^{4}$, no significant difference could be determined in this case $\left(p_{H_{0}}=0.2685\right)^{5}$. Thus, a significant improvement of the gas source tracing performance in terms of the average path length cannot be asserted. However, it is important to note that the tested reference strategy, which approximately implements specular reflection at the walls, does not provide uniform coverage over a designated area [8]. Instead, the central area is covered excessively at the expense of the periphery [26]. Hence, the fact that the Braitenberg vehicle does not outperform the reference strategy might be caused by the inherent tendency of the reference strategy to "find" a gas source in the middle of the testbed area by default.

\footnotetext{
${ }^{4}$ Because the path lengths are not normally distributed, a statistical test was performed that does not assume a specific distribution.

${ }^{5}$ The Wilcoxon two sample test evaluates the hypothesis $H_{0}$ that the populations from which the samples are taken have identical median values.
} 


\begin{tabular}{|c|cccc|ccc|}
\hline Source & Strategy & $K_{v}\left[\frac{\mathrm{cm}}{\mathrm{s}}\right]$ & Trials & $l_{\text {tot }}[\mathrm{m}]$ & $\bar{l}[\mathrm{~m}]$ & $\bar{d}[\mathbf{c m}]$ & $\bar{v} / K_{v}$ \\
\hline \multirow{5}{*}{ Corner } & Ref $(1-\mathrm{x})$ & 5 & 76 & 1554.9 & $20.46 \pm 19.38$ & $223.5 \pm 78.7$ & $97.6 \%$ \\
& PL, 1-x & $5 / 3$ & 107 & 1251.1 & $11.69 \pm 11.22$ & $199.3 \pm 88.2$ & $75.5 \%$ \\
& PL, 1-x & 5 & 52 & 733.3 & $14.10 \pm 14.56$ & $217.8 \pm 90.1$ & $81.2 \%$ \\
& PL, 1-x & 3 & 55 & 517.8 & $9.42 \pm 6.84$ & $183.8 \pm 84.9$ & $71.3 \%$ \\
\hline
\end{tabular}

Table 2. Statistics of the gas source tracing experiments with a Braitenberg vehicle and the source placed in a corner of the testbed area. Comparison of the "permanent love" behaviour (PL) with the reference strategy (Ref). If different speed gains $K_{v}$ were tested, both of them are given separated by a slash.

Additional tests were therefore performed with the source placed at a less prominent location near to one corner of the field $(15 \mathrm{~cm}$ away from the beginning of the repellent wall potential along both the $\mathrm{x}$ - and $\mathrm{y}$-axis). A typical run is shown in Fig. 6. The trajectory corresponds nicely with the concentration gridmap. After the robot got "caught" by the plume-like structure, it drove very slowly towards the source following an almost straight line. As in the experiments with the gas source placed in the middle, however, the trajectory often cannot be explained by the time-averaged structure of the distribution alone.

The results of the experiments with the gas source placed in a corner of the testbed area are summarised in Table 2. For each corner a total of approximately 3 hours of trials was performed both with and without a gas source. The average path length needed to reach an active gas source was $11.69 \mathrm{~m}$, compared to $20.46 \mathrm{~m}$ in the reference experiments (median: $7.25 \mathrm{~m}$ compared to $17.09 \mathrm{~m}$ ). In contrast to the situation where the gas source was placed in the middle of the testbed area, the Wilcoxon two samples test reveals a highly significant improvement in tracing performance in terms of the average path length $\left(p_{H_{0}} \ll 0.01\right)$.

This result clearly shows that following the instantaneous concentration gradient can improve the gas source tracing performance compared to search strategies that do not consider cues from the sensed gas distribution. Note that a better performance in this context means that the robot reaches the source along a shorter path and not necessarily in shorter time. This follows from the dependency of the increase in path length performance, $l_{\text {tot }}$, on the applied speed gain $K_{v}$, which is a consequence of the non-zero response time of the metal oxide gas sensors. Although this conclusion cannot be drawn with high statistical certainty from the experiments with $K_{v}=5 \mathrm{~cm} / \mathrm{s}$ and $K_{v}=3 \mathrm{~cm} / \mathrm{s}\left(p_{H_{0}}=0.9727\right)$, it is apparent that a Braitenberg-type strategy, which relies on measurements that reflect the stimulus with a certain delay, should perform less well if the speed gain $K_{v}$ increases. Thus, a standard search strategy will perform better in terms of time if it operates at sufficiently high speed. In fact even at the rather low speeds investigated, the reference strategy performs comparably well in this respect: the average time to reach the source was $419.3 \mathrm{~s}$ for the reference search, compared to $348.6 \mathrm{~s}\left(K_{v}=5 \mathrm{~cm} / \mathrm{s}\right)$ and $444.1 \mathrm{~s}$ $\left(K_{v}=3 \mathrm{~cm} / \mathrm{s}\right)$ for the Braitenberg vehicles. Thus, the reference strategy can outperform both the "permanent love" behaviours tested in terms of the time needed to reach the gas source, if a speed gain of approximately $K_{v}>6 \mathrm{~cm} / \mathrm{s}$ is applied. 

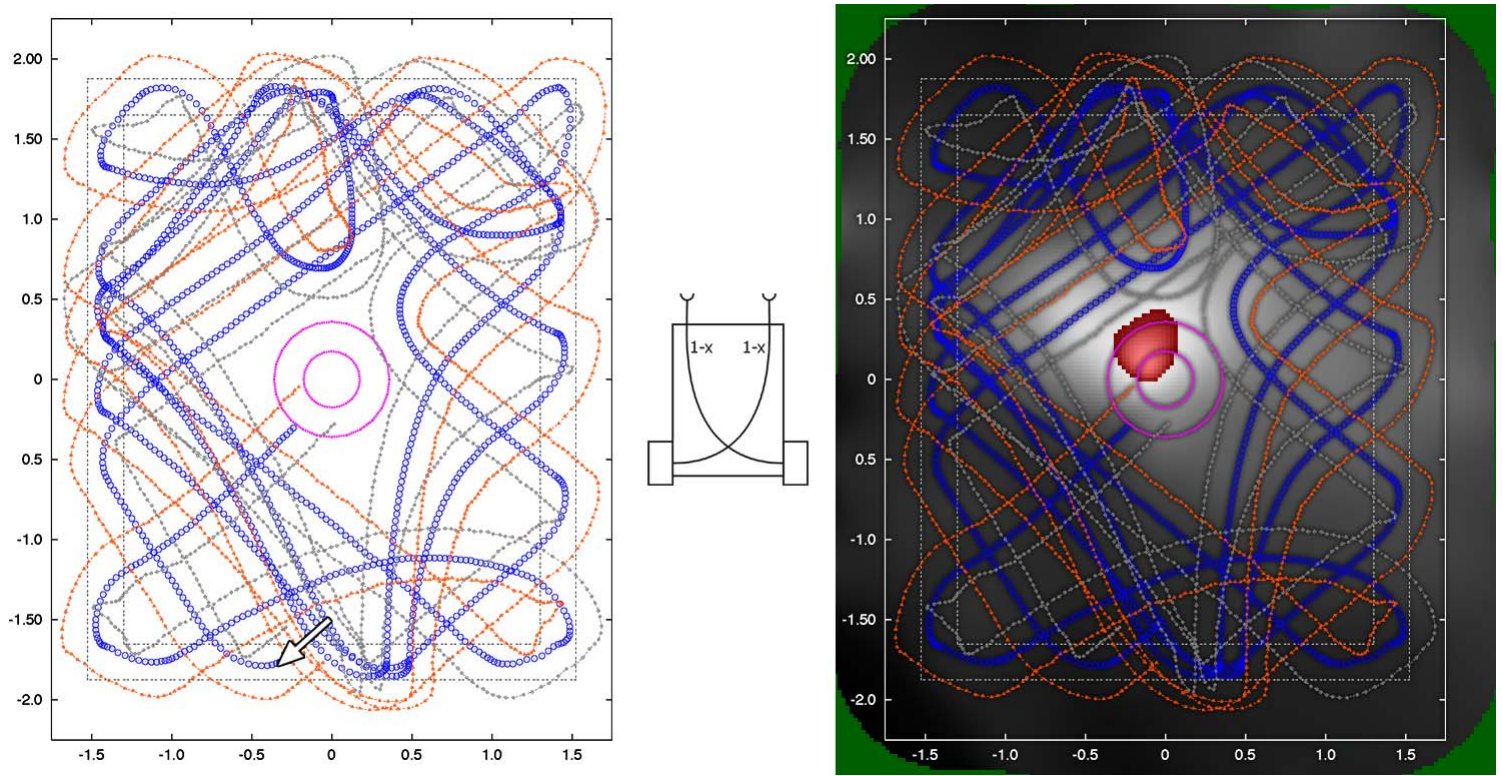

Fig. 7. Example of the path of a Braitenberg-Vehicle with crosssed (1-x)-connections ("exploring love") with the gas source in the middle of the testbed area.

\subsection{Localisation by Exploration and Concentration Peak Avoidance}

With crossed connections a completely different behaviour results (see Table 3). Although the robot is expected to stay near the source - again assuming a smooth gradient - and thus collisions should not be unlikely, the robot managed to avoid the source most of the time (see Fig. 7). The difference compared to the trials with uncrossed connections is apparent and can be demonstrated with high confidence by a Wilcoxon two sample test $\left(p_{H_{0}}<0.01\right)$.

Though this strategy is obviously not suitable for driving the robot towards a gas source as quickly as possible, it offers an interesting solution to the full gas source localisation problem, including the declaration that the source has been found. This can be seen in Fig. 8, which shows the complete path of the robot during the experiment, of which one trial (out of five) is shown in Fig. 7. After combining the paths of all the trials in this experiment, the location of the source is indicated by the part of the picture (Fig. 8, left)

\begin{tabular}{|c|cccc|ccc|}
\hline Source & Strategy & $K_{v}\left[\frac{\mathrm{cm}}{\mathrm{s}}\right]$ & Trials & $l_{\text {tot }}[\mathbf{m}]$ & $\bar{l}[\mathbf{m}]$ & $\bar{d}[\mathbf{c m}]$ & $\bar{v} / K_{v}$ \\
\hline \multirow{3}{*}{ Middle } & Ref $(1-\mathrm{x})$ & 5 & 33 & 319.0 & $9.67 \pm 7.66$ & $136.7 \pm 44.9$ & $95.9 \%$ \\
& PL, 1-x & 5 & 123 & 1044.0 & $8.49 \pm 7.93$ & $127.4 \pm 45.7$ & $73.5 \%$ \\
& EL, 1-x & $5 / 3$ & 18 & 731.8 & $40.66 \pm 34.10$ & $143.3 \pm 41.0$ & $76.5 \%$ \\
\hline
\end{tabular}

Table 3. Statistics of the gas source tracing experiments with a Braitenberg vehicle and the source placed in the middle of the testbed area. Comparison of the "permanent love" behaviour (PL) with the "exploring love" behaviour (EL) and the reference strategy (Ref). If different speed gains $K_{v}$ were tested, both of them are given separated by a slash. 

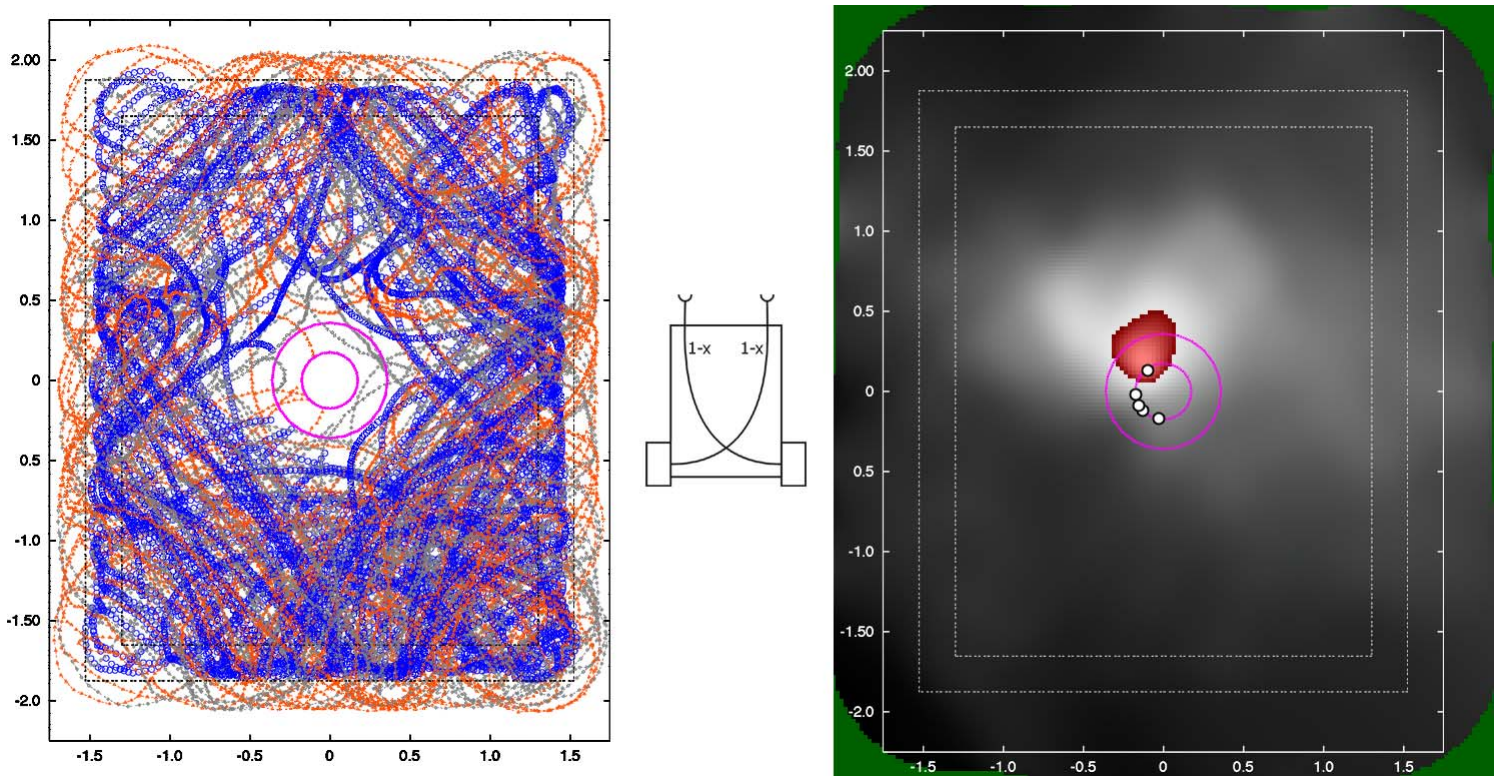

Fig. 8. Left side: path of a Braitenberg-Vehicle with crosssed (1-x)-connections ("exploring love") during a complete experiment, lasting 3 hours and including 5 separate trials. Right side: Concentration gridmap determined from the readings collected during the same experiment. The locations where the robot entered the clearance area around the gas source are indicated by small circles.

that remained uncovered by the plotted trajectory, i.e., the central area that was not crossed by the robot. Notice that in contrast to the area that is covered by an obstacle (the inner circle in Fig. 8), the area between the concentric circles should not remain uncovered in the case of a dummy obstacle (no active gas source).

The reason why exploration and concentration peak avoidance might be a good solution for gas source localisation can be explained as follows: with crossed inhibitory connections the robot explores the available space and evades each local concentration maximum. Because there exist many of them, it is hard to find a particular maximum that belongs to the actual source by a hillclimbing strategy. On the other hand, concentration maxima occur more frequently near the gas source and thus the source's location remains comparatively uncovered in a plot of the robot trajectory such as Fig. 8, left.

Note that the experiments point to a feature that can be used to identify a gas source rather than providing a complete solution that is generally applicable. If the source is not detectable as an obstacle, for example, it would not be possible to avoid collisions with the currently used setup. During the experiments the robot occasionally moved into the clearance area around the gas source. As can be seen on the right side of Fig. 8, which shows the position where the robot entered the clearance area, this happened mostly if the robot approached the gas source from the opposite side of the plume-like structure (an example of such a collision is shown in Fig. 7). A collision with the gas source could corrupt the practicability of the proposed method if the robot spilled the smelling liquid or wetted its tyres, which would cause the robot to become a gas source too. 


\section{Conclusions and Future Work}

This article is concerned with the problem of tracing and declaring a static gas source by a mobile robot using electrochemical gas sensors. Two Braitenberg-type strategies were investigated in an environment without an artificially controlled airflow. In a total of 36.5 hours of experiments where the robot drove almost 5 kilometers, both strategies were shown to be of possible use for gas source localisation.

Using uncrossed inhibitory connections it was found that the average path length the robot needs to move to the source can be decreased. The path length could be reduced by up to a factor of two compared to a strategy that explores the available area ignoring the gas sensor readings. This factor, however, depends on the considered situation and the reference search strategy used.

For real world applications this strategy has to be extended by an additional declaration mechanism to determine that the gas source has been found with high certainty. A method based on concentration measurements only, which requires the robot to perform a rotation maneouvre in front of a possible gas source, was introduced and experimentally verified in [23]. Further mechanisms for gas source declaration could be added by using other sensors, which provide clues on possible sources, for example, by recognising a beaker or a puddle of the analyte by vision.

With crossed connections the robot evades each local concentration maximum including those that are closest to the source. Due to the fact that maxima occur more frequently near the gas source, the path of the robot covers the area near to the actual location of the source less densely compared to distant regions.

Applying this strategy therefore offers a possible solution to the task of gas source declaration without using additional sensors. A further advantage is that the average distance to the gas source is increased (see Tables $1-3$ ) and that direct contact between the robot and the volatile or liquid substance to be detected can be diminished. This might be preferable in the case of an analyte that is corrosive or in other ways offensive to the robot itself. Furthermore, it helps to prevent the robot from wetting its wheels in cases where the gas source is a liquid substance that cannot be sensed as an obstacle. This is generally not desired because the robot would thereby become a gas source too. With the design of the Mark III mobile nose, however, it was not possible to avoid collisions with the gas source completely. Future work should therefore address possible modifications to achieve a collision-free path even if the gas source cannot be sensed as an obstacle. A suggestion would be to add a third gas sensor array in a tube that sticks out to the front of the robot in order to examine the area the robot is about to drive towards. If this third tube protruded over the robot's front sufficiently, it should be possible to decrease the probability of the robot crossing a puddle of the analyte, even if the robot approached the source from a down-wind direction.

A possible objection to the suggested method is time consumption. Because the actual location of a gas source is determined by excluding all other possible locations, the time needed to locate the source increases with the size of the area observed. On the other hand, the time consumption would scale down with the number of robots utilised. And after all, 
there is as yet no other experimentally verified method to localise a gas source that does not appear as an obstacle to the robot.

Acknowledgments This work was sponsored with a Marie Curie grant that was part of the European Commission's 5th framework programme (FP5), which is gratefully acknowledged by the first named author. All the small problems that arose during the preparation for the described experiments could be fastly fixed due to the kind help of Alexander Skoglund, Per Sporrong, Kevin LeBlanc and Grzegorz Cielniak. Finally the fruitful discussions with Amy Loutfi and Boyko Iliev greatly helped with all the remaining experimental and theoretical problems. Thanks to all these helpful colleagues.

\section{References}

1. N. Barsan and A. Tomescu. Calibration Procedure for SnO2-based Gas Sensors. Thin Solid Films, 259:91 95, 1995.

2. V. Braitenberg. Vehicles: Experiments in Synthetic Psychology. MIT Press/Bradford Books, 1984.

3. C. David, J. Kennedy, J. Ludlow, and J. Perry. A Re-Appraisal of Insect Flight Towards a Point Source of Wind-Borne Odor. Journal of Chemical Ecology, 8:1207-1215, 1982.

4. T. Duckett, M. Axelsson, and A. Saffiotti. Learning to Locate an Odour Source with a Mobile Robot. In Proceedings of the IEEE International Conference on Robotics and Automation (ICRA 2001), Seoul, South Korea, May, 21-26 2001.

5. J. F. Engelberger. Robotics in Service. Kogan Page, London, 1989.

6. A. M. Farah and T. Duckett. Reactive Localisation of an Odour Source by a Learning Mobile Robot. In Proceedings of the Second Swedish Workshop on Autonomous Robotics, pages 29-38, Stockholm, Sweden, October 10-11 2002.

7. G. Fraenkel and D. Gunn. The Orientation of Animals. Clarendon Press, Oxford, 1940.

8. D. Gage. Randomized Search Strategies with Imperfect Sensors. In Proceedings of SPIE Mobile Robots VIII, volume 2058, pages 270-279, 1993.

9. J. W. Gardner and P. Bartlett. A Brief History of Electronic Noses. Sensors and Actuators B, 18-19:211-220, 1994.

10. A. Hayes, A. Martinoli, and R.M.Goodman. Swarm Robotic Odor Localization. In Proceedings of the 2001 IEEE/RSJ International Conference on Intelligent Robots and Systems (IROS-01), volume 2, pages 1073-1078, Maui, Hawaii, USA, October 2001.

11. J. O. Hinze. Turbulence. McGraw-Hill, New York, 1975.

12. O. Holland and D. McFarland. Artificial Ethology. Oxford University Press, New York, 2001.

13. H. Ishida, Y. Kagawa, T. Nakamoto, and T. Moriizumi. Odour-Source Localization in the Clean Room by an Autonomous Mobile Sensing System. Sensors and Actuators B, 33:115-121, 1996.

14. H. Ishida, K. Suetsugu, T. Nakamoto, and T. Moriizumi. Study of Autonomous Mobile Sensing System for Localization of Odor Source Using Gas Sensors and Anemometric Sensors. Sensors and Actuators A, 45:153$157,1994$.

15. H. Ishida, M. Tsuruno, K. Yoshikawa, and T. Moriizumi. Spherical Gas-Sensor Array for Three-Dimensional Plume Tracking. In Proceedings of the IEEE International Conference on Advanced Robotics (ICAR 2003), pages 369-374, Coimbra, Portugal, 2003.

16. O. Khatib. Real-Time Obstacle Avoidance for Manipulators and Mobile Robots. In Proceedings of the IEEE International Conference on Robotics and Automation (ICRA 1985), pages 500-505, 1985.

17. J. C. Latombe. Robot Motion Planning. Kluwer Academic, 1991.

18. A. Lilienthal and T. Duckett. A Stereo Electronic Nose for a Mobile Inspection Robot. In Proceedings of the IEEE International Workshop on Robotic Sensing (ROSE 2003), Örebro, Sweden, 2003.

19. A. Lilienthal and T. Duckett. An Absolute Positioning System for 100 Euros. In Proceedings of the IEEE International Workshop on Robotic Sensing (ROSE 2003), Örebro, Sweden, 2003.

20. A. Lilienthal and T. Duckett. Creating Gas Concentration Gridmaps with a Mobile Robot. In Proceedings of the 2003 IEEE/RSJ International Conference on Intelligent Robots and Systems (IROS 2003), pages 118 123, Las Vegas, USA, 2003. 
21. A. Lilienthal and T. Duckett. Experimental Analysis of Smelling Braitenberg Vehicles. In Proceedings of the IEEE International Conference on Advanced Robotics (ICAR 2003), pages 375-380, Coimbra, Portugal, 2003.

22. A. Lilienthal and T. Duckett. Gas Source Localisation by Constructing Concentration Gridmaps with a Mobile Robot. In Proceedings of the European Conference on Mobile Robots (ECMR 2003), pages 159 - 164, Warsawa, Poland, 2003.

23. A. Lilienthal, H. Ulmer, H. Fröhlich, A. Stützle, F. Werner, and A. Zell. Gas Source Declaration with a Mobile Robot. In Proceedings of the IEEE International Conference on Robotics and Automation (ICRA 2004), pages $1430-1435,2004$.

24. A. Lilienthal, A. Zell, M. R. Wandel, and U. Weimar. Experiences Using Gas Sensors on an Autonomous Mobile Robot. In Proceedings of EUROBOT 2001, 4th European Workshop on Advanced Mobile Robots, pages 1-8. IEEE Computer Press, 2001.

25. A. Lilienthal, A. Zell, M. R. Wandel, and U. Weimar. Sensing Odour Sources in Indoor Environments Without a Constant Airflow by a Mobile Robot. In Proceedings of the IEEE International Conference on Robotics and Automation (ICRA 2001), pages 4005-4010, 2001.

26. M. J. McNish. Effects of uniform target density on random search. Master's thesis, Naval Postgraduate School, Monterey, California, 1987.

27. T. Nakamoto, H. Ishida, and T. Moriizumi. A Sensing System for Odor Plumes. Analytical Chem. News 8 Features, 1:531-537, August 1999.

28. R. A. Russell. Ant Trails - an Example for Robots to Follow? In IEEE Int Conf. Robotics and Automation (ICRA 1999), pages 2698-2703, 1999.

29. R. A. Russell. Odour Sensing for Mobile Robots. World Scientific, 1999.

30. R. A. Russell, L. Kleeman, and S. Kennedy. Using Volatile Chemicals to Help Locate Targets in Complex Environments. In Proceedings of the Australian Conference on Robotics and Automation, pages 87-91, Melbourne, Aug 30- Sept 12000.

31. R. A. Russell and A. H. Purnamadjaja. Odour and Airflow: Complementary Senses for a Humanoid Robot. In IEEE Int Conf. Robotics and Automation (ICRA 2002), pages 1842-1847, 2002.

32. R. A. Russell, D. Thiel, R. Deveza, and A. Mackay-Sim. A Robotic System to Locate Hazardous Chemical Leaks. In IEEE Int Conf. Robotics and Automation (ICRA 1995), pages 556-561, 1995.

33. R. A. Russell, D. Thiel, and A. Mackay-Sim. Sensing Odour Trails for Mobile Robot Navigation. In IEEE Int Conf. Robotics and Automation (ICRA 1994), pages 2672-2677, 1994.

34. T. Sharpe and B. Webb. Simulated and Situated Models of Chemical Trail Following in Ants. In R. Pfeifer, B. Blumberg, J.-A. Meyer, and S. Wilson, editors, Proceedings of the 5th Conference on Simulation of Adaptive Behaviour, pages 195-204, 1998.

35. E. Stella, F. Musio, L. Vasanelli, and A. Distante. Goal-oriented Mobile Robot Navigation Using an Odour Sensor. In Proceedings of the Intelligent Vehicles Symposium '95, pages 147-151, 1995.

36. M. R. Wandel, A. Lilienthal, T. Duckett, U. Weimar, and A. Zell. Gas Distribution in Unventilated Indoor Environments Inspected by a Mobile Robot. In Proceedings of the IEEE International Conference on Advanced Robotics (ICAR 2003), pages 507-512, Coimbra, Portugal, 2003. 